\title{
Physical mechanism of temperature oscillations in ferroelectric ceramics conduction
}

\author{
Ya.I. Lepikh \\ Scientific \& Technical Center "Phonon", Special Design \& Technological Bureau "Element", 29 Prospect Acad. Glushko, Odessa, \\ 65104, Ukraine \\ Tel.: (0482) 66-82-29; Fax: (0482) 47-02-23
}

\begin{abstract}
For ferroelectric ceramics of the lead zirconate-titanate PZT-5 system the results of investigations of conduction temperature oscillations are presented. An explanation for the effect of conduction temperature oscillations is given. It is shown that the effect is due to relaxation of the perovskite-type $90^{\circ}$-domains and action of mobile charged point defects. The proposed physical mechanism is confirmed using the acoustic emission technique. Some propositions concerning use of this effect in the instrument making are formulated.
\end{abstract}

Keywords: polarization vector, domain, charge, oscillations, relaxation, ferroelectric, temperature.

Paper received 25.01.00; revised manuscript received 12.05.00; accepted for publication 06.06.00.

1. It was reported in [1] that, when studying temperature dependence of conduction in ferroelectric ceramics of the lead zirconate-titanate PZT-5 system, an effect of temperature oscillations of conduction has been found. Periodic (with frequency of about $10 \mathrm{~Hz}$ ) resistance oscillations were observed near the 80,110 and $140{ }^{\circ} \mathrm{C}$ temperature points (Fig. 1). The amplitude of these oscillations was maximal at $140{ }^{\circ} \mathrm{C}$. The effect was observed far from the phase transition in piezoelectric ceramics (Curie point $T_{c}=360{ }^{\circ} \mathrm{C}$ ) and remained stable during numerous heating-cooling cycles. No explanation has been given, however, for this effect, as well as to similar anomalies in temperature dependence of conduction in layered $\mathrm{A}^{\mathrm{III}} \mathrm{B}^{\mathrm{III}} \mathrm{C}_{2}^{\mathrm{VI}}$-type ferroelectrics (observed in [2]) and ceramics based on doped titanium-containing oxides [3].

2. In this paper an explanation is given for the effect of conduction temperature oscillations in PZT-5 ferroelectric ceramics. The explanation is based on further studies that followed assumptions made in [1]. Essentially, it is as follows.

In the context of the macroscopic order-disorder concept, ferroelectric ceramics belonging to the lead zirconate-titanate system is of perovskite-type. Such a material actually has a domain structure with domain boundaries, point defects, micro- and macrostrains and local stresses. Of particular importance in ferroelectrics is the macroscopic orientation order-disorder. It is expressed by spontaneous polarization vector orientations of 180 and $90^{\circ}$ domains. A diagram of ferroelectric crystal fragments is presented in Fig. 2.

During polarization under action of external electric field (strength of $5 \mathrm{kV} / \mathrm{mm}$ ) and heating (temperature of $140^{\circ} \mathrm{C}$ ) the ferroelectric domain structure is removed from the steady equilibrium and transformed (Fig. 2b). In this case rotation of a $180^{\circ}$-domain (whose spontaneous polarization vector is opposite to the external electric field $E$ ) occurs without mechanical motion. It is realized by interchanging positive and negative charges of a dipole cell-domain. Such a rotation of $180^{\circ}$ domains (even if they grow by gaining adjacent domains) does not result in geometric size change for the sample studied and induces no intrinsic stresses.

Concurrent with this, mobile charged point defects move to the ferroelectric surface under action of external electric field. There they and the bound charges of $180^{\circ}$ domains form intrinsic electric field of ferroelectric. Besides, moving point defects upset balance of electric forces near the $90^{\circ}$-domain boundary, thus promoting its motion.

The $90^{\circ}$ domains are rigid dipoles whose polarization vector $P$ makes an angle $\theta$ with the electric field $E$. The magnitude of torque $M$ acting on a domain in the 


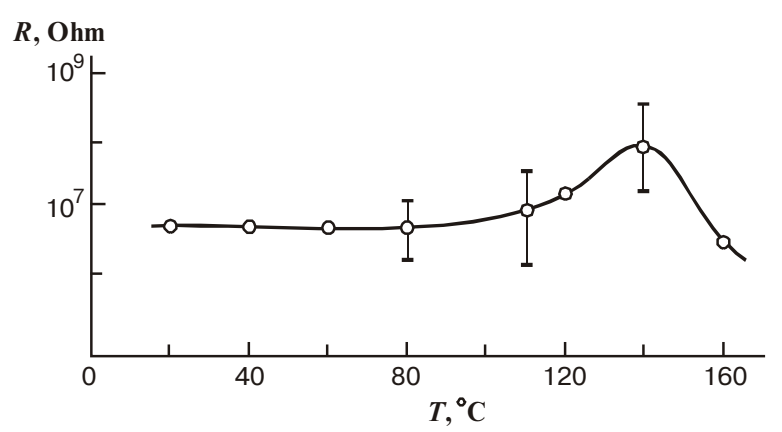

Fig.1. Temperature dependence of ferroelectric ceramics resistance.

electric field is $2 E q l \sin \theta$ (here $q$ is the charge and $l$ is the spacing between the centers of charge masses; it approximately equals to the domain size). When the external electric field exceeds the coercive field, the $90^{\circ}$ domains rotate in such a way as to make vector $P$ parallel to vector $E$ (Fig. 2b).

3. Generally the above motion is not quiet even in a uniform external electric field, because a domain has to overcome the potential wells (produced by point defects) and friction of neighboring domains. In addition, thermal atomic motion has an effect. Thermal fluctuations affect the stability of the domain polarization vector at any non-zero temperature. They make this vector oscillate about its stable equilibrium.

However, a new position of a $90^{\circ}$ domain that it holds as a result of rotation is unstable. When the external electric field is withdrawn, the elastic rotation forces (which grow with domain rotation) are counterbalanced by a static electric field produced by redistributed charges of $180^{\circ}$ domains and charged point defects. Instability of such a state leads to $90^{\circ}$ domain relaxation in more strong external fields of any nature (electric, thermal, etc.). Under certain conditions (degree of instability) existing in our case their backward motion to the position where thermodynamic potential is minimal is rather intense. As a result, the $90^{\circ}$-domains pass the position of stable equilibrium. When they move back, they again pass this position because they get some energy from external thermal field. That is how oscillations of the spontaneous polarization vector occur.

Temporal change in ferroelectric polarization leads to appearance of electric current due to motion of bound charges. The current density is $J=d P / d t$. This results in the temperature oscillations of ferroelectric ceramics conduction that has been found by us in PZT-5. The current value $I$ is determined by the number $n$ of domains involved in oscillations. If all the domains are of the same size, then $I=n(d P / d t)$.

4. The concept of our physical model for temperature oscillations of ferroelectric ceramics conduction corresponds to experimental data obtained by Little and Fawsek (see [4]). They have found that motion of $90^{\circ}$ domain boundaries is of relaxation type and essentially depends on point defects. However, a direct confirmation of the physical mechanism for temperature oscillations proposed in this work makes a very difficult problem which requires to consider processes of domain boundaries motion and interaction with point defects on an atomic scale. In addition, the actual piezoelectric ceramic materials (composites) have both micro- and macroscopic nonuniformities. They are characteristic of a heterophase structure in which domains of the ordered phase are built in a disordered matrix, and domain sizes may substantially vary over the sample bulk.

That is why we used the acoustic emission technique to provide support for the proposed oscillation mecha-
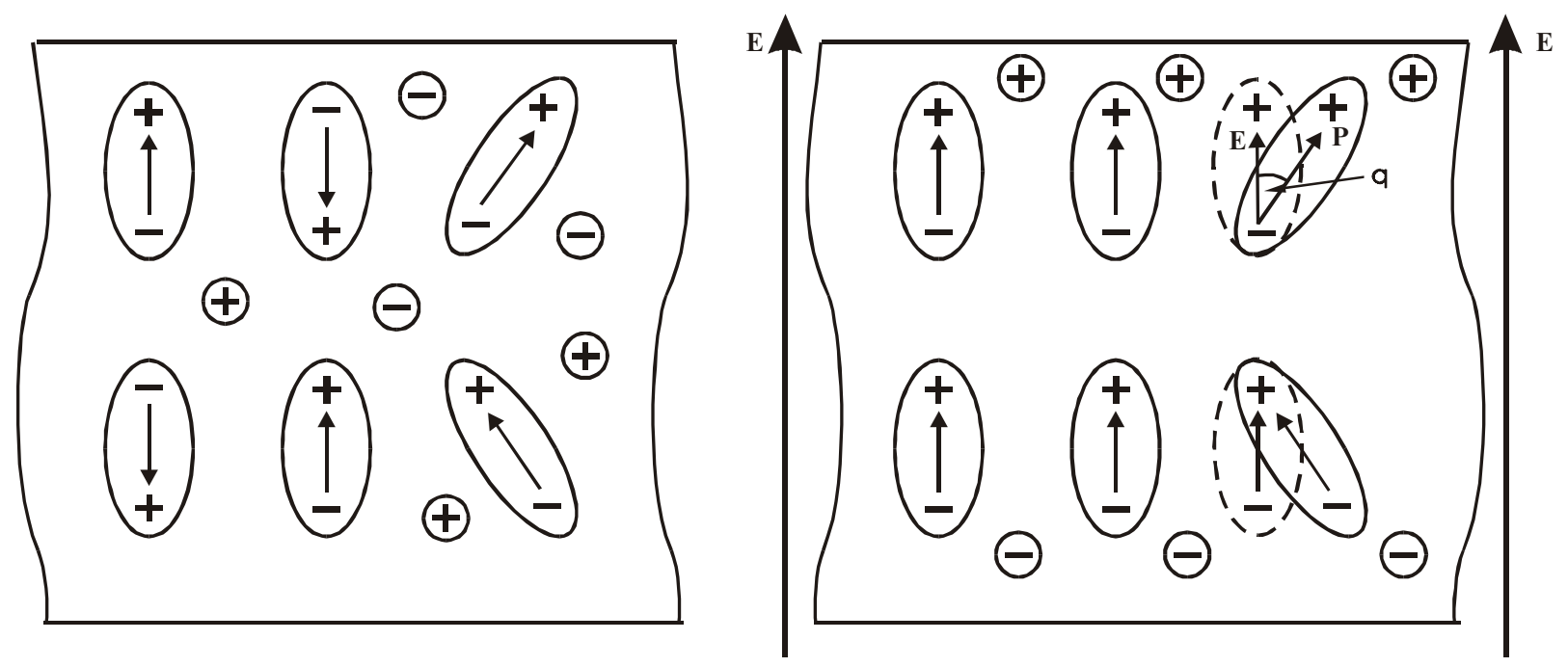

Fig. 2. A diagram of ferroelectric structure fragment without (a) and with (b) external electric field; $\oplus, \ominus-$ point defects,
domains. 
Ya.A. Lepikh: Physical mechanism for temperature oscillations of ferroelectric ...

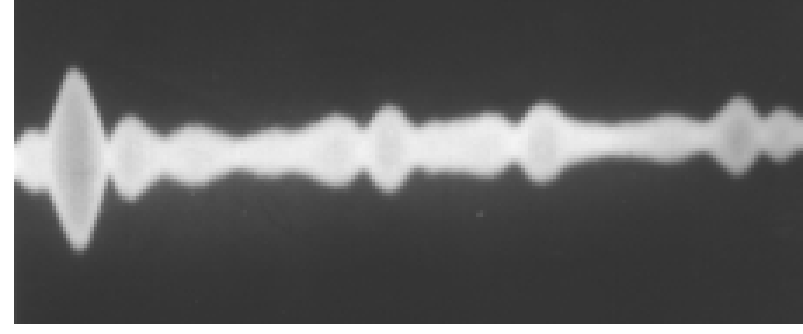

Fig.3. Oscillogram of acoustic emission signals at temperature oscillations of ferroelectric ceramics conduction.

nism. It was expected that transformation of the inner structure of ferroelectric ceramics should be accompanied with the corresponding acoustic signals. The studies were carried out using the technique given in [5]. Our experimental investigations enabled us to detect signals of acoustic emission near $140{ }^{\circ} \mathrm{C}$. These signals had a rise of frequency spectrum near the lower boundary of the range of audibility. An oscillogram of acoustic emission signals, including those reflected from the sample boundaries, is presented in Fig. 3. No such signals were detected at 80 and $110^{\circ} \mathrm{C}$ temperature points. One could state, following [6], that at the above points the total number of acts of acoustic signal generation is rather small (below 150), and their energy dissipation during propagation is too essential, to record experimentally the structural processes in the material studied.

\section{Conclusions}

Thus the results of our experimental investigations of acoustic emission lend support to the proposed physical model for the effect of temperature oscillations of conduction in PZT-5 ferroelectric ceramics.

The thermal oscillation effect may be used in the instrument making, temperature control equipment, control systems for technological processes. It seems possible to fabricate a thermal relay-generator. Its simplest design might be a bridge circuit with such a thermal element providing unbalance of the Wheatstone bridge arm when parameters of the temperature field change.

\section{References}

1. Ya.I. Lepikh, Temperature oscillations of conduction in piezoelectric ceramics (in Russian) // Pisma v ZhTF 22(4), pp.65-77 (1996).

2. V.A. Aliev, N.Z. Gasanov, G.D. Guseinov, Conductivity behavior in phase transition region of layered $\mathrm{A}^{\mathrm{III}} \mathrm{B}^{\mathrm{III}} \mathrm{C}_{2}^{\mathrm{VI}}$ type ferroelectrics (in Russian), in Abstracts XII All-Union Conf. on Physics of Ferroelectrics (Rostov-on-Don), 2, p.136 (1989).

3. V.A. Ganin, M.A. Kvantov, On the nature of nonlinear properties of semiconductor ceramics based on doped titaniumcontaining oxides, in Abstracts XII All-Union Conf. on Physics of Ferroelectrics (Rostov-on-Don), 2, p.137 (1989).

4. M. Lynes, A. Glass, Ferroelectrics and Related Materials (Russian translation), Mir, Moscow (1981).

5. V.A. Goreshnikov, Yu.B. Drobot, Acoustic Emission. Application for Testing Materials and Products (in Russian), Izdatel'stvo Standartov, Moscow (1976).

6. R. Truell, C. Elbaum, B.B. Chick, Ultrasonic Methods in Solid State Physics, Academic Press, New York and London (1969). 